03.5

\title{
Статистический анализ формирования пузыря Тейлора в капиллярной трубке
}

\author{
(С) Ф.В. Роньшин ${ }^{1,2}$, Д.Ю. Кочкин ${ }^{1,2}$, Ю.А. Дементьев ${ }^{1,2}$, К.С. Элоян $^{1,2}$, И.С. Вожаков ${ }^{1,2}$ \\ ${ }^{1}$ Новосибирский государственный университет, Новосибирск, Россия \\ ${ }^{2}$ Институт теплофизики им. С.С. Кутателадзе СО РАН, Новосибирск, Россия \\ E-mail: f.ronshin@gmail.com
}

Поступило в Редакцию 10 декабря 2021 г.

В окончательной редакции 10 января 2022 .

Принято к публикации 11 января 2022 г.

\begin{abstract}
Выполнено экспериментальное исследование режима Тейлора в трубке с внутренним диаметром $2 \mathrm{~mm}$. Разработан и впервые применен метод исследования режима Тейлора в капиллярной трубке с использованием автоматического анализа изображений для измерения основных характеристик пузырей. Исследованы зависимости длин пузырей и жидкостных перемычек от скоростей газа и жидкости. Обнаружено, что в области стабильного режима Тейлора среднеквадратичное отклонение размеров пузырей близко к точности метода исследования. При приближении к границам режима дисперсия распределения размеров пузырей возрастает. Показано, что на основании статистического анализа большого объема данных можно сделать вывод о наличии слияния и дробления пузырей.
\end{abstract}

Ключевые слова: капиллярная трубка, двухфазный поток, режим Тейлора, статистический анализ.

DOI: 10.21883/PJTF.2022.06.52210.19105

За последние два десятилетия использование микрореакторов стало одним из важных методов интенсификации процессов для химической и перерабатывающей промышленности [1] благодаря эффективности тепломассопереноса, актуальной для химического производства. Потенциал применения микрореакторов был продемонстрирован во многих областях, таких как смешивание [2], разделение [3], реакции [4] и химический анализ [5]. В мини-каналах снарядный режим течения, или режим Тейлора, является одним из основных режимов течения, который реализуется в широком диапазоне расходов газа и жидкости [6]. В режиме Тейлора поток состоит из длинных пузырьков, разделенных жидкими пробками. Пузыри Тейлора окружены тонкой пленкой жидкости и имеют длину, превышающую характерный диаметр канала. Фундаментальные данные о таком режиме течения получены для каналов различной геометрии. В некоторых случаях требуется дополнительная информации о стабильности режима. В фармацевтической промышленности пузыри Тейлора используются для получения малых доз лекарства, а в химической - для смешивания веществ в определенной пропорции с большой точностью, синтеза наночастиц [7] и катализируемых газожидкостных реакций [8]. В таких случаях отклонение в размере пузырей должно предсказываться и контролироваться. В настоящей работе с использованием автоматизированного алгоритма проведен статистический анализ длин пузырей Тейлора на основе большого объема экспериментальных данных.

Для исследования режима Тейлора был разработан и собран рабочий участок (рис. 1). Для визуализации использовалась стеклянная трубка с внутренним диамет- ром $2 \mathrm{~mm}$ и длиной $12 \mathrm{~cm}$. Ко входу трубки подключен Т-образный смеситель. Жидкость (вода Milli-Q®) подавалась при помощи шприцевого жидкостного насоса Cole-Parmerß, газ (азот) подавался из баллона, расход

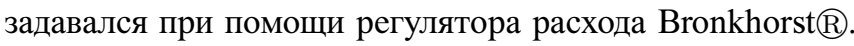
Обработка сигналов с расходомера, а также контроль расхода газа осуществлялись с помощью аналогоцифрового преобразователя National Instruments $($. Чистота рабочей жидкости контролировалась путем измерения поверхностного натяжения жидкости тензиометром KRUSS K100. Визуализация двухфазного течения в трубке осуществлялась с помощью высокоскоростной камеры Vision Research Phantom® v.7.0 со скоростью съемки $1000 \mathrm{fps}$. Область камеры захватывала часть трубки длиной $29 \mathrm{~mm}$ с пространственным разрешением 40 px/mm. Приведенная скорость газа $U_{S G}$, определяемая как расход газа, деленный на площадь поперечного сечения трубы, составляла от 0.1 до $0.6 \mathrm{~m} / \mathrm{s}$, а жидкости $U_{S L}$ - от 0.05 до $0.3 \mathrm{~m} / \mathrm{s}$.

Для автоматической обработки данных по визуализации пузырей при тейлоровском режиме течения и получения количественных характеристик разработан алгоритм в среде Matlab®. Автоматизированный метод анализа изображений хорошо зарекомендовал себя при обработке изображений в мини- и микросистемах [9], а также при исследовании динамики роста пузыря при кипении [10]. В программу подаются изображения, записанные в ходе эксперимента на скоростную камеру Phantom 囚. Далее происходит анализ изображения, определяются области, соответствующие газовым пузырькам и жидкостным перемычкам. Затем происходит анализ пузырей (их положения, размеров и других параметров). 


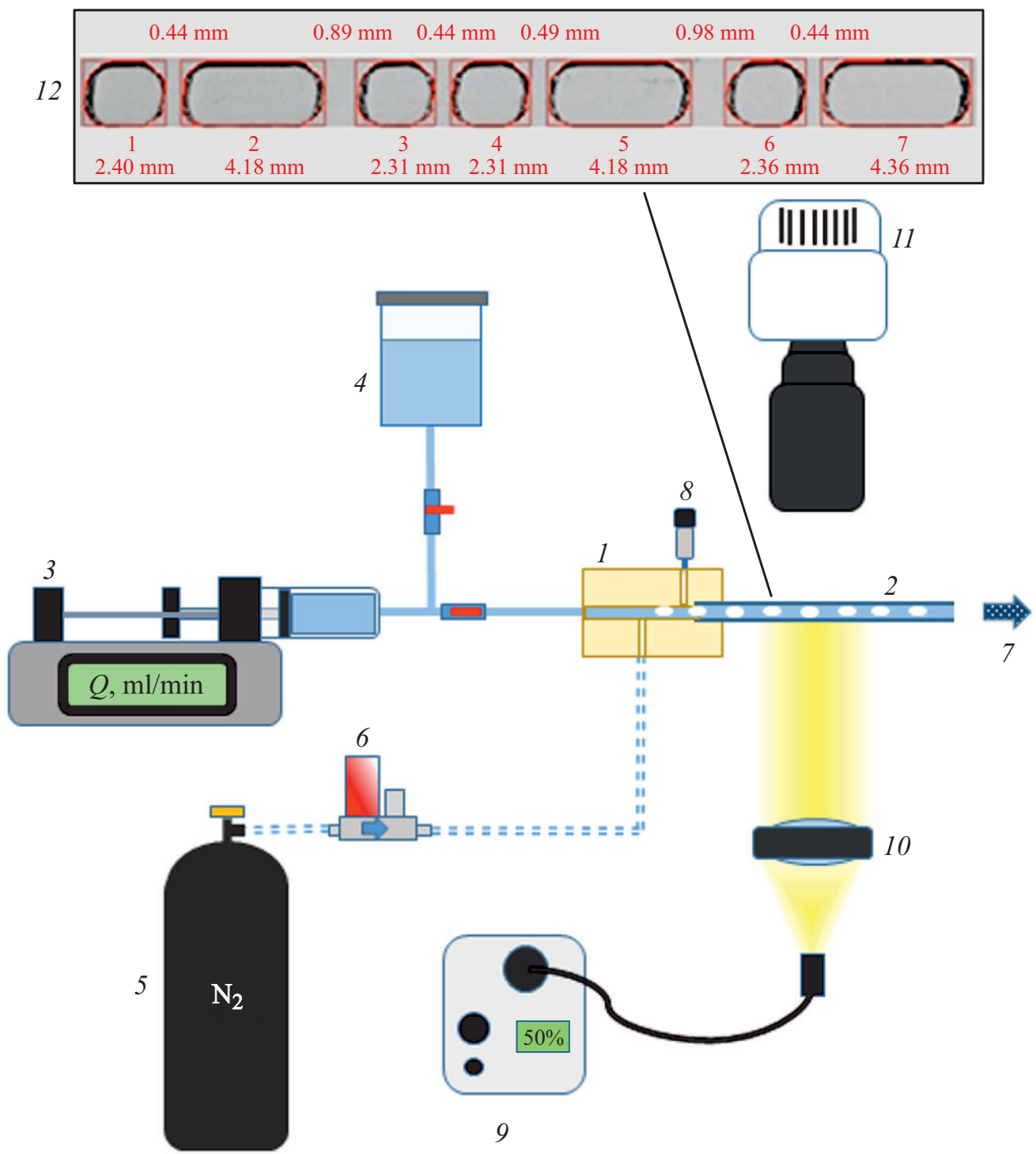

Рис. 1. Экспериментальный стенд по исследованию двухфазного течения в круглом канале и пример обработки с помощью разработанного алгоритма. 1 - Т-смеситель, $2-$ рабочий участок, 3 - шприцевой жидкостный насос, $4-$ емкость с водой Milli-Q, 5 - баллон с азотом, 6 - регулятор расхода, 7 - выход в атмосферу, 8 - датчик давления, 9 - источник света, $10-$ линза, 11 - высокоскоростная камера, 12 - обработанное с помощью автоматического алгоритма изображение.

Такой же анализ проводится для следующего кадра. Таким образом, осуществляется анализ эволюции каждого пузыря во времени, что позволяет определить скорость пузырей. В итоге данные по размеру и скорости фиксируются для каждого пузыря. Подобный анализ проводится и для жидкостных перемычек. Точность измерений определяется неоднозначностью определения межфазной границы газ-жидкость, которая составляет 1 рх или $0.025 \mathrm{~mm}$.

На рис. 1 показано характерное обработанное изображение. Каждый из пузырей пронумерован, и программа отслеживает эволюцию во времени. Также для каждого пузыря приведены длина и длина жидкостной перемычки. Для каждого значения расходов газа и жидкости анализируется 3000 кадров. Программа записывает в файл значения размеров пузырей, их скоростей, длин жидкостных перемычек и т.д. для каждого кадра.

На рис. 2 представлено распределение длин пузырей в зависимости от приведенных скоростей газа и жидкости. При небольших приведенных скоростях жидкости и газа $\left(U_{S L}=0.1 \mathrm{~m} / \mathrm{s}, U_{S G}=0.1 \mathrm{~m} / \mathrm{s}\right)$ длины пузырей различаются несущественно. Так, среднее значение длины пузырей $\left\langle L_{b}\right\rangle$ составляет $1.92 \mathrm{~mm}$, а среднеквадратичное отклонение $\sigma$ равно $0.03 \mathrm{~mm}$, что сопоставимо с точностью измерений (размер выборки 2674 пузыря). При увеличении приведенной скорости жидкости до $U_{S L}=0.2 \mathrm{~m} / \mathrm{s}$ средний размер пузырей уменьшается до $1.52 \mathrm{~mm}$, в то время как среднеквадратичное отклонение остается неизменным (размер выборки 364 пузыря). При увеличении приведенной скорости газа 

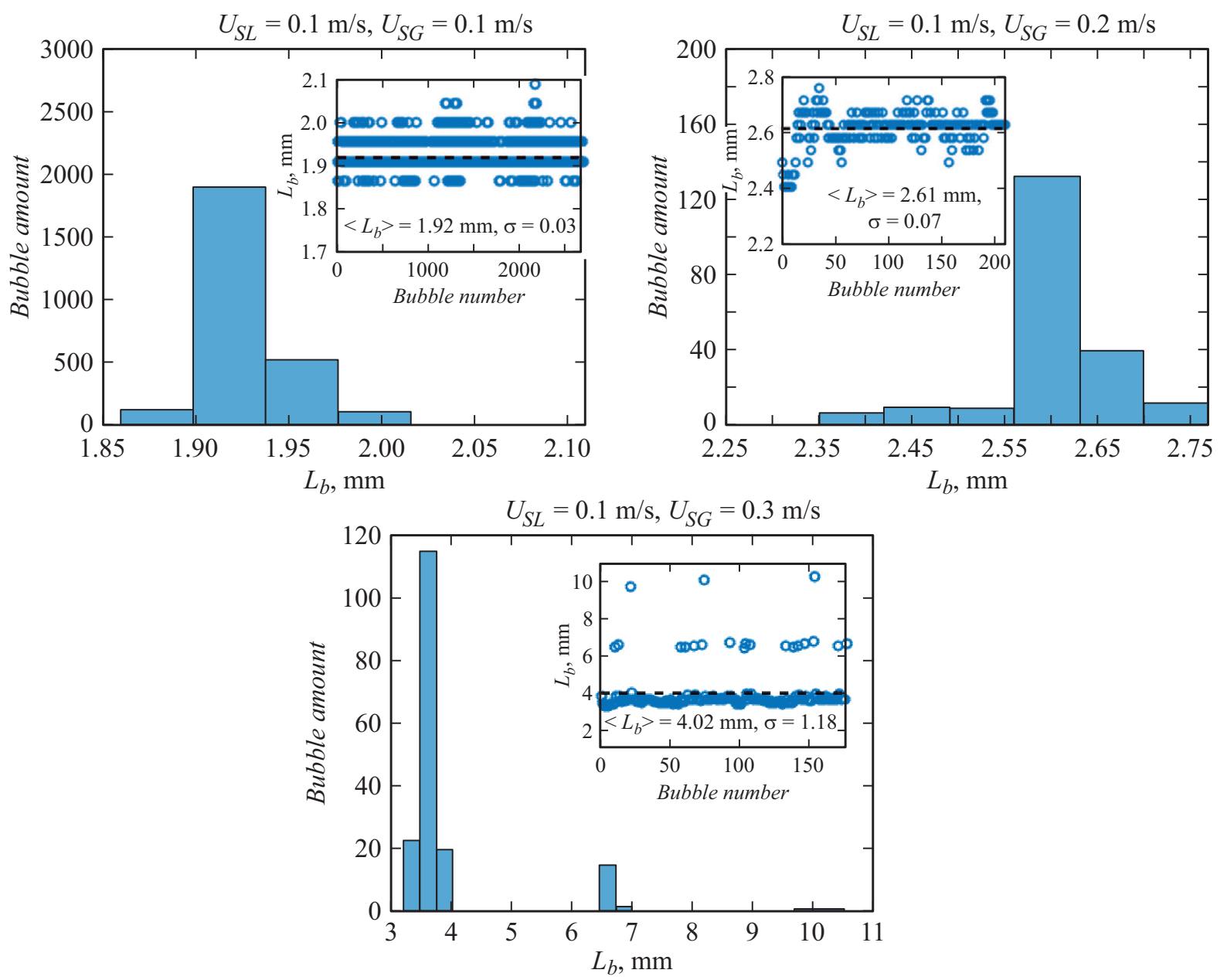

Рис. 2. Распределения длин пузырей в зависимости от приведенных скоростей газа и жидкости.

до $U_{S G}=0.2 \mathrm{~m} / \mathrm{s}\left(U_{S L}=0.1 \mathrm{~m} / \mathrm{s}\right)$ среднее значение $\left\langle L_{b}\right\rangle$ увеличивается до $2.61 \mathrm{~mm}$, а среднеквадратичное отклонение $\sigma$ возрастает в 2.5 раза до значения $0.07 \mathrm{~mm}$ (размер выборки 210 пузырей). При дальнейшем увеличении скорости газа $\left(U_{S G}=0.3 \mathrm{~m} / \mathrm{s}\right)$ и приближении к границам режима Тейлора начинается коалесценция пузырей. При этом в канале наблюдается некоторый характерный размер пузырей (около 90\% всех пузырей), а также размеры, кратные характерному, которые сформированы вследствие слияния нескольких пузырей. В этом случае более информативным является не среднее значение $\left\langle L_{b}\right\rangle=4.02 \mathrm{~mm}$, а медианное $L_{b}=3.64 \mathrm{~mm}$. Среднеквадратичное отклонение в случае коалесценции резко возрастает до значения $1.18 \mathrm{~mm}$ (размер выборки 178 пузырей), однако если исключить образовавшиеся в результате коалесценции пузыри, то эта величина составит $0.13 \mathrm{~mm}$.

При еще большем увеличении скорости газа наблюдаются переходные режимы: доли коалесцирующих и начальных пузырей становятся сопоставимы, причем вероятность каскадной коалесценции существенно возрастает, а размеры наблюдаемых пузырей могут различаться в 10 раз. Минимальные размеры пузырей для различных скоростей газа $U_{S G}$ показаны на рис. 3, $a$. В диапазоне скоростей $0.1-0.5 \mathrm{~m} / \mathrm{s}$ наблюдается плавный рост минимального размера пузырьков, а при скорости $0.6 \mathrm{~m} / \mathrm{s}$ - резкое падение. По всей видимости, уменьшение минимального размера связано с наличием дробления длинных пузырей, которые образованы в результате каскадной коалесценции.

На рис. 3, $а$ представлена зависимость размера газовых пузырей $L_{b}$ и жидкостных перемычек $L_{s}$ от приведенной скорости газа $U_{S G}$. Можно видеть, что размер пузырей увеличивается с увеличением приведенной скорости газа. До скорости газа $0.5 \mathrm{~m} / \mathrm{s}$ рост происходит плавно. Вследствие коалесценции периодически наблюдаются пузыри кратного размера, но это не вносит существенного вклада в структуру потока. При более высоких значениях приведенной скорости газа происходит резкое увеличение средней длины пузырей. Это обусловлено существенным повышением вероятности коалесценции, что подтверждается анализом распределения по длинам пузырей. Сплошной линией на рисунке показана корреляция [11]: $L_{b}=\left(1+0.57 U_{S G} / U_{S L}\right) D$. Экспериментальные значения лежат несколько ниже представленной зависимости. Выбивается только точка, в 


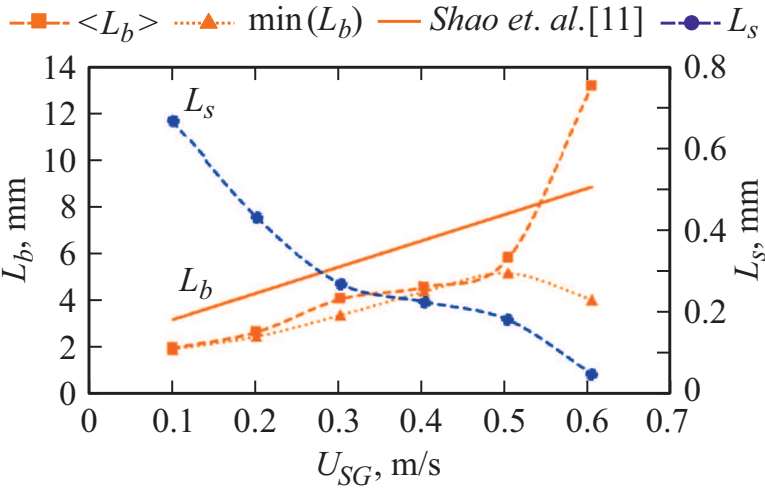

$b$

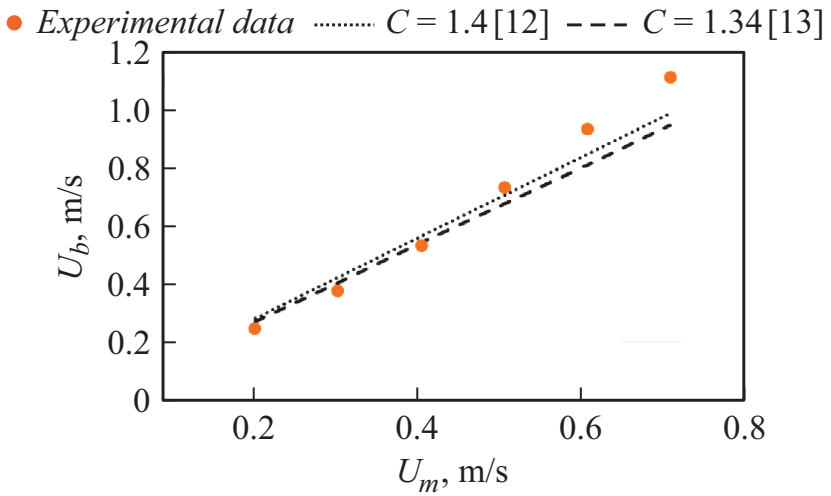

Рис. 3. $a-$ зависимость длины газовых пузырей и жидкостных перемычек от приведенной скорости газа при $U_{S L}=0.1 \mathrm{~m} / \mathrm{s} . b-$ зависимость скорости газовых пузырей от скорости смеси при $U_{S L}=0.1 \mathrm{~m} / \mathrm{s}$.

которой коалесценция оказывает существенное влияние на распределение размеров пузырей.

На рис. 3, $b$ представлена зависимость скорости пузырей от скорости смеси $U_{m}$, определяемой как сумма приведенных скоростей газа и жидкости. Можно видеть, что скорость пузырей монотонно увеличивается с увеличением приведенной скорости смеси. Влияние параметров межфазного распределения в потоке определяется параметром распределения $C$. Для горизонтальных труб скорость пузыря определяется произведением $U_{b}=C U_{m}$. Из рис. $3, b$ видно хорошее согласие экспериментальных данных с корреляциями для $C=1.4$ [12] и 1.34 [13].

Таким образом, нами разработан и собран рабочий участок для исследования режима Тейлора в круглой трубе диаметром $2 \mathrm{~mm}$ с Т-образным смесителем. Для обработки экспериментальных данных по визуализации и получения количественных характеристик разработан и впервые применен алгоритм, анализирующий эволюцию пузырей и жидкостных перемычек во времени, а также позволяющий определить их размер и скорость в капиллярной трубке. Показано, что при небольших приведенных скоростях газа $U_{S G}=0.1 \mathrm{~m} / \mathrm{s}$ и жидкости $U_{S L}=0.1 \mathrm{~m} / \mathrm{s}$ длины пузырей слабо отличаются от среднего значения, а среднеквадратичное отклонение сопоставимо с ошибкой метода исследования. Увеличение скорости жидкости не приводит к увеличению абсолютного значения разброса размеров пузырей, но уменьшает их средний размер. Увеличение приведенной скорости газа ведет к существенному росту отклонения размеров пузырей от среднего значения. Так, при увеличении скорости газа до $U_{S G}=0.2 \mathrm{~m} / \mathrm{s}$ среднеквадратичное отклонение возрастает в 2.5 раза. При увеличении скорости газа и приближении к границам режима Тейлора наблюдается коалесценция пузырей. Это приводит к появлению кратных пиков в распределении пузырей по размерам и резкому (на порядок) росту среднеквадратичного отклонения. Кроме того, в этом случае средний размер пузырей существенно отличается от медианного значения. Так, при скоростях газа $U_{S G}=0.3 \mathrm{~m} / \mathrm{s}$ и жид- кости $U_{S L}=0.1 \mathrm{~m} / \mathrm{s}$ различие этих размеров составило около 10\%. При еще большем увеличении скорости газа наблюдается переходный режим, в котором не существует выделенного размера пузырей, а минимальный и максимальный размеры могут различаться в 10 раз, что является следствием как каскадной коалесценции, так и дробления пузырьков.

\section{Финансирование работы}

Исследование выполнено за счет гранта Российского научного фонда (проект № 20-79-10096).

\section{Конфликт интересов}

Авторы заявляют, что у них нет конфликта интересов.

\section{Список литературы}

[1] M.N. Kashid, L. Kiwi-Minsker, Ind. Eng. Chem. Res., 48, 6465 (2009). DOI: 10.1021/ie8017912

[2] J. Hou, G. Qian, X. Zhou, Chem. Eng. J., 167, 475 (2011). DOI: $10.1016 /$ j.cej.2010.10.054

[3] N. Oozeki, S. Ookawara, K. Ogawa, P. Löb, V. Hessel, AlChE J., 55, 24 (2009). DOI: 10.1002/aic.11650

[4] P. Lang, M. Hill, I. Krossing, P. Woias, Chem. Eng. J., 179, 330 (2012). DOI: 10.1016/j.cej.2011.11.015

[5] E. Livak-Dahl, I. Sinn, M. Burns, Ann. Rev. Chem. Biomol. Eng., 2, 325 (2011).

DOI: 10.1146/annurev-chembioeng-061010-114215

[6] А.В. Минаков, А.А. Щебелева, А.А. Ягодницына, А.В. Ковалев, А.В. Бильский, Письма в ЖТФ, 43 (18), 82 (2017). DOI: 10.21883/PJTF.2017.18.45037.16549 [A.V. Minakov, A.A. Shebeleva, A.A. Yagodnitsyna, A.V. Kovalev, A.V. Bilsky, Tech. Phys. Lett., 43 (9), 857 (2017). DOI: 10.1134/S1063785017090231].

[7] A. Günther, S.A. Khan, M. Thalmann, F. Trachsel, K.F. Jensen, Lab Chip, Iss. 4, 278 (2004). DOI: 10.1039/B403982C

[8] T. Yasukawa, W. Ninomiya, K. Ooyachi, N. Aoki, K. Mae, Chem. Eng. J., 167, 527 (2011). DOI: $10.1016 /$ j.cej.2010.08.077 
[9] F. Ronshin, Y. Dementyev, D. Kochkin, K. Eloyan, I. Vozhakov, Exp. Therm. Fluid Sci., 132, 110565 (2022). DOI: 10.1016/j.expthermflusci.2021.110565

[10] V. Serdyukov, I. Malakhov, A. Surtaev, J. Visualization, 23, 873 (2020). DOI: 10.1007/s12650-020-00660-z

[11] N. Shao, W. Salman, A. Gavriilidis, P. Angeli, Int. J. Heat Fluid Flow., 29, 1603 (2008).

DOI: 10.1016/j.ijheatfluidflow.2008.06.010

[12] D.J. Nicklin, Chem. Eng. Sci., 17, 693 (1962).

DOI: 10.1016/0009-2509(62)85027-1

[13] K. Mishima, T. Hibiki, H. Nishihara, Int. J. Multiphase Flow, 19, 115 (1993). DOI: 10.1016/0301-9322(93)90027-R 